\title{
Social transmission of egg rejection in a cuckoo host
}

\author{
Canchao Yang $^{1 *}$, William E Feeney ${ }^{2,3^{*}}$
}

3

$4 \quad{ }^{1}$ Ministry of Education Key Laboratory for Ecology of Tropical Islands, College of Life

$5 \quad$ Sciences, Hainan Normal University, Haikou 571158, China.

$6 \quad{ }^{2}$ Environmental Futures Research Institute, Griffith University, Nathan, Australia

$7 \quad{ }^{3}$ Department of Behavioural Ecology and Evolutionary Genetics, Max Planck Institute for

8 Ornithology, Seewiesen, Germany

$9 \quad$ * Correspondence to: ccyang@hainnu.edu.cn (C.Y.), william.e.feeney@gmail.com (W.E.F.)

11 Abstract

12 Social learning can enable the rapid dissemination of behaviors throughout a population.

13 Rejection of foreign eggs is a key defense in hosts of avian brood parasites; however,

14 whether social cues can inform whether a host rejects an egg remains unknown. Here, we

15 aimed to determine whether access to social information can influence egg rejection behavior

16 in semi-colonial barn swallows (Hirundo rustica). By manipulating the social information

17 available from a neighboring nest, we found that swallows that had access to social

18 information (i.e. neighbor recently rejected an egg) were more likely to reject a foreign egg

19 compared to those that did not have access to social information (i.e. neighbor did not reject

20 an egg). This result provides the first empirical evidence that egg rejection behavior can

21 solely be informed by social information, and in doing so highlights the dynamic nature of

22 defenses that hosts can deploy against brood parasitism. 


\section{Introduction}

26 Species interactions are important ecological processes that shape and regulate biodiversity

27 (Thompson 1999). Phenotypes that underpin an individual's ability to survive and reproduce

28 can emerge and spread throughout a population via several processes, including those that

29 refine over generations (e.g. morphological characteristics) and are passed on through genetic

30 inheritance (Phillips \& Shine 2004; Spottiswoode \& Stevens 2012), and those that emerge

31 rapidly (e.g. behavioral innovations) and can be spread throughout a population through

32 social transmission (Thornton \& Raihani 2008; Davies \& Welbergen 2009; Aplin et al.

33 2015). The tractable "arms-races" that emerge between avian brood parasites and hosts has

34 contributed to their use as model systems to study the ecology and evolution of species

35 interactions (Feeney et al. 2014; Soler 2014). Unlike most birds, brood parasites lay their

36 eggs in the nests of other birds and foist the burden of parental care onto the host. The cost of

37 brood parasitism selects reciprocal defensive adaptations in hosts and offensive adaptations in

38 parasites that span all stages of the host's nesting cycle (Brooke \& Davies 1988; Langmore et

39 al. 2003; De Marsico et al. 2012; Feeney et al. 2012).

40

41 Of the various defenses that hosts deploy against brood parasitism, rejection of foreign eggs

42 is best studied (Soler 2014). The decision to reject an egg is enabled through well-defined

43 behavioral "rules", such as rejecting the odd egg out (the 'discordancy hypothesis') or

44 rejecting any egg (or eggs) that do not match a learned or innate internal template ('true

45 recognition') (Rothstein 1974; Lotem et al. 1995; Hauber \& Sherman 2001; Stevens et al.

46 2013). The decision to reject an egg can be further influenced by personal information about

47 a host's perceived risk of parasitism (Davies et al. 1996; Feeney et al. 2015), and once egg

48 rejection emerges in a population it can persist despite generations of allopatry with brood

49 parasites (Lahti 2006; Hale \& Briskie 2007; Peer et al. 2011). However, despite evidence of 
50 social transmission of information being important for other defenses against brood

51 parasitism, such as recognition and mobbing of adult parasites (Davies \& Welbergen 2009;

52 Feeney \& Langmore 2013), and suspicion that the decision to reject an egg may too be

53 impacted by social information (Soler 2011), empirical evidence that egg rejection can be

54 influenced by social information is lacking.

56 Here, we used the barn swallow (Hirundo rustica) to investigate whether egg rejection

57 behaviors are influenced by access to social information. In China, barn swallows are

58 occasionally parasitized by the common cuckoo (Cuculus canorus), with a parasitism rate of

59 0-2.4\% (Yang et al. 2015; Su et al. 2017), and they are capable of recognizing and grasp-

60 ejecting non-mimetic parasite eggs at a rate of 24-63\% (Yang et al. 2015). They are semi-

61 colonial (i.e. females nest between 0.5 - 10m from their closest neighbor) and build open

62 cup-shaped nests, permitting the possible visual transmission of egg rejection between

63 individuals (Møller 2002). The high rejection rate and low natural parasitism rate currently

64 experienced by barn swallows in China, as well as their open-cup nests and semi-coloniality,

65 makes them a well-suited model organism to investigate whether egg rejection can be

66 influenced solely by social information.

\section{Results and Discussion}

69 In 2018, we first conducted a series of egg rejection trials using a blue egg $(n=55$, common

70 cuckoos often lay blue eggs in China (Fossøy et al. 2016)), conspecific swallow egg ( $n=48)$

71 and no egg ( $n=43$, during these trials experimenter behavior at the nest was identical but no

72 egg was added) and found that swallows rejected $61.82 \%$ of blue eggs, with $85.29 \%$ of

73 rejections occurring within 5 h. No eggs were rejected (i.e. ejected or deserted) during the

74 conspecific and no egg trials. Then, in 2019, swallow nests were experimentally set up in 
75 pairs $3 \mathrm{~m}$ apart with nest entrances facing one another (Fig. 1A). Once colonized, a blue egg

76 was placed in one nest (partner nest) from each pair and the behavioral response after $5 \mathrm{~h}$ was

77 used to determine whether the nest it was paired with (focal nest) would subsequently be used

78 for a "transmission" trial (if the egg was rejected) or "non-transmission" trial (if the egg was

79 not rejected). Nest pairs designated for control trials experienced comparable disturbance to

80 the transmission and non-transmission nests, but no egg was added. Following this, the focal

81 nest received a blue egg during early incubation to investigate whether access to social

82 information increased the likelihood of the swallow from the focal nest rejecting the foreign

83 egg.

85 Our results indicate that barn swallows that have access to social information are more likely

86 to reject a foreign egg, and do so more quickly than those that have not had access to social

87 information. We found that there was a significant effect of treatment on egg rejection rates

88 (GLMM: $F_{2,137}=4.855, P=0.009$; Fig. $1 B$ ): rejection of blue eggs was significantly higher in

89 the transmission $(87.5 \%, n=56)$ versus non-transmission $(59.62 \%, N=52)$ (Sequential

90 Bonferroni: $P=0.004)$ trials, and we found no difference in rejection rates between the non-

91 transmission and the control trials $(60.98 \%, n=41)$ (Sequential Bonferroni: $P=0.839)$. We

92 also found no significant effects of clutch size (GLMM: $\left.F_{4,137}=0.767, P=0.549\right)$ or incubation

93 day (i.e. the number of days after incubation commenced that the trial began) (GLMM:

$\left.94 \mathrm{~F}_{5,137}=0.409, P=0.842\right)$. We found that time until rejection was significantly predicted by

95 treatment (Cox regression: Wald $=10.847, \mathrm{df}=2, P=0.004)$, with blue eggs more likely to be

96 rejected within $1 \mathrm{~h}$ during the transmission trial (60.71\% of rejected eggs) compared to the

97 non-transmission and control trials, which were more likely to rejected within $1-3 \mathrm{~h}(13.46 \%$

98 and $7.32 \%$, respectively) (Fig. 1C). Again, we found no effect of clutch size (Cox regression: 
99 Wald $=1.980, \mathrm{df}=4, P=0.739)$ or incubation day (Cox regression: Wald=2.986, $\mathrm{df}=5, P=0.702)$

100 on the time until rejection across treatments.

101

102 These data indicate that hosts can mediate egg rejection behaviors solely based off access to

103 social information. It is well-established that defenses that operate to prevent parasitism from

104 occurring, such as recognizing and responding to the threat post by an adult parasite near a

105 nest, are learned behaviors (Davies \& Welbergen 2009; Langmore et al. 2012; Feeney \&

106 Langmore 2013) that wane in the absence of parasitism (Briskie et al. 1992; Hobson \&

107 Villard 1998; Lindholm \& Thomas 2000; Hale \& Briskie 2007). Likewise, personal

108 information about the perceived risk of parasitism can affect the likelihood of hosts rejecting

109 a foreign egg (Davies et al. 1996; Feeney et al. 2015; Thorogood \& Davies 2016) or chick

110 (Langmore et al. 2009). During the two years over which this study was conducted no natural

111 parasitism was recorded, only blue model eggs were rejected, and the only significant

112 predictor of increased egg rejection was having a neighbor that previously rejected an egg.

113 This suggests that in addition to being highly sensitive to information about the presence of

114 cuckoos in the area, hosts can also be sensitive to parasite-specific behaviors in conspecifics,

115 to the extent that they can make egg rejection decisions solely using this information. The

116 nuanced use of both personal and social information by hosts has implications for

117 coevolutionary trajectories in these systems, potentially underpinning a dynamic mosaic of

118 phenotypically plastic defenses across host populations that parasites must counter, which

119 may outpace and shape genetic evolution.

120

121 Materials and Methods

122

\section{Study area and species}


124 In this study, we used a barn swallow (Hirundo rustica) population in the Jinping District of

125 southeast China ( $23^{\circ} 352$ ' $-24^{\circ} 401^{\prime} \mathrm{N}, 116^{\circ} 658^{\prime}-116^{\circ} 706^{\prime}$ E) to examine whether egg

126 rejection behaviors could be influenced by social information. Barn swallows are

127 insectivorous, semi-colonial passerines that build mud nests on walls under eaves and

128 typically nest within $0.5-10 \mathrm{~m}$ of their nearest conspecific neighbour. They are occasionally

129 parasitized by the common cuckoo (Cuculus canorus), with a parasitism rate of 0-2.4\%. Barn

130 swallows are capable of recognizing and grasp-ejecting non-mimetic parasite eggs, with a

131 rejection rate of 24-63\% (Yang et al. 2015). All experiments in this study were conducted

132 between March-May of 2018 and 2019.

\section{Experimental procedure}

135 In 2018, we tested the egg rejection rate of the barn swallow population for non-mimetic and 136 conspecific eggs using artificial parasitism experiments. For the non-mimetic ("parasite")

137 trials, we used blue model eggs that were made of polymer clay and similar in size to barn 138 swallow eggs. The model eggs were blue because while the egg phenotype of common 139 cuckoos that parasitise barn swallows in China is not known, common cuckoos often lay blue 140 eggs in China (Fossøy et al. 2016). In each trial, one blue model egg was inserted into each 141 swallow nest on the day following clutch completion. The artificially parasitized nests were 142 checked at $1 \mathrm{hr}, 3 \mathrm{hr}, 5 \mathrm{hr}, 24 \mathrm{hr}, 72 \mathrm{hr}$, and $144 \mathrm{hr}$ after egg placement (6 days in total). In the 143 conspecific trial, the experimental procedure was identical, except that a conspecific egg 144 from another nest was added to the host nest instead of a model egg. All conspecific eggs 145 were returned to the appropriate nest after the experiment. A control treatment, which 146 followed identical experimental procedures except that no egg was added, was performed to 147 control for the effects of disturbance and egg manipulation. We found that the egg rejection 148 rate for this population of the blue eggs was $61.82 \%$, with $85.29 \%$ of the rejections occurring 
149 within 5 hr ( $n=55)$. All conspecific eggs were accepted by hosts $(n=48)$, and no desertions or

150 ejections were observed in any trials, including the control trial $(n=43)$. The period from

151 spanning the beginning of egg incubation to the end of nestling feeding was approximately 15238 days.

154 In 2019, we experimentally manipulated the social transmission of egg rejection using the 155 same barn swallow population. Swallow nests were collected before the breeding season (late 156 February) and glued to walls in pairs. The paired nests were $3 \mathrm{~m}$ apart, with the nest entrances 157 facing each other (Fig. 1A). Each pair of nests was at least 30m from the next closest pair. To 158 ensure that nests were not resampled during the experiment, we required that the nesting 159 period of all pairs included in the study were conducted within a 38-day period. Within each 160 pair of nests, one was considered the focal nest, and the other nest was considered the partner 161 nest.

163 During the experimental trials, one blue model egg (identical to those used in 2018) was 164 inserted into the partner nest during the early incubation period (i.e. within six days of clutch 165 completion) between 6:30-7:30am. To control for the effects of nest manipulation, the 166 partner nests in the control trials were manipulated, but no eggs were added. To avoid host 167 observation of artificial parasitism, a black umbrella was used as a shield during egg transfers 168 and nest manipulation. After 5hr, the experimental nests were checked; we chose to check the nests after $5 \mathrm{hr}$ because most $(85.29 \%)$ of the rejections in 2018 occurred within $5 \mathrm{hr}$.

171 If the egg was not rejected from the partner nest within $5 \mathrm{hr}$, the model egg was removed and

172 the pair of nests was assigned to the non-transmission trial. If the egg was rejected from the 173 partner nest within 5hr, we assigned the pair of nests to the transmission trial. Following this, 
174 a blue model egg was inserted into the focal nests of all pairs assigned to the non-

175 transmission, transmission, and control trials (Fig. 1). The focal nests in all three trials were

176 checked $1 \mathrm{hr}, 3 \mathrm{hr}, 5 \mathrm{hr}, 24 \mathrm{hr}, 72 \mathrm{hr}$, and $144 \mathrm{hr}$ after artificial parasitism. The control trial was

177 used to control for the effects of the partner nest on rejection behavior. The focal host

178 response to the model egg was classified as rejection if the parasite egg was ejected or

179 deserted, and as acceptance if the parasite egg continued to be incubated. We predicted that

180 the egg rejection rate in the focal nest would be higher in transmission trial because this

181 individual had previously had access to social information of egg rejection from the partner

182 nest. We also predicted that the egg rejection rats of the focal nests would be similar between

183 the non-transmission and control treatments, as these individuals had not previously had

184 access to social information.

\section{Statistical analyses}

187 A generalized linear mixed model (GLMM) was used to quantify the effects of artificial 188 parasitism on egg rejection among trials. In this model, the rejection or acceptance of the 189 parasite egg was set as the response variable; the three trials (non-transmission, transmission, 190 and control), clutch size, and incubation day (i.e. the number of days after incubation 191 commenced that the trial began) were set as fixed effects; and nest identity and date of first 192 egg laying date were set as random effects. We compared pairs of significant factors using 193 Sequential Bonferroni tests. Cox regressions, which calculate survival probabilities at given 194 time points, were used to compare the speed of egg rejection among trials. That is, we 195 evaluated the probability that the parasite egg had not yet been rejected at each time point 196 (1hr, 3hr, 5hr, 24hr, 72hr, and 144hr) for each trial. Thus, this analysis compared how quickly 197 the hosts in different trials recognized the parasite egg. All statistical analyses were 198 performed using IBM SPSS 25.0 for Windows (IBM Inc., USA). 


\section{ACKNOWLEDGEMENTS}

201 This work was funded by the Natural Science Foundation of China (No. 31672303) (CY) and

202 the Alexander von Humboldt Foundation (WEF).

203

204 AUTHOR CONTRIBUTIONS

205 CY conceived the idea, with assistance from WEF. CY collected and analyzed the data. Both 206 authors wrote the manuscript.

207

\section{COMPETING INTERESTS}

209 The authors declare no competing interests.

\section{DATA AVAILABILITY}

212 All data used in this manuscript is provided in the supplementary materials (Supplemental 213 File 1).

\section{REFERENCES}

Aplin, L.M., Farine, D.R., Morand-Ferron, J., Cockburn, A., Thornton, A. \& Sheldon, B.C.

217 (2015). Experimentally induced innovations lead to persistent culture via conformity

218 in wild birds. Nature, 518, 538-541.

219 Briskie, J.V., Sealy, S.G. \& Hobson, K.A. (1992). Behavioral defenses against avian brood

220 parasitism in sympatric and allopatric host populations. Evolution, 46, 334-340.

221 Brooke, M. de L. \& Davies, N.B. (1988). Egg mimicry by cuckoos Cuculus canorus in

222 relation to discrimination by hosts. Nature, 335, 630-632. 
223 Davies, N.B., Brooke, M.D.L. \& Kacelnik, A. (1996). Recognition errors and probability of

224 parasitism determine whether reed warblers should accept or reject mimetic cuckoo 225 eggs. Proc. R. Soc. B-Biol. Sci., 263, 925-931.

226 Davies, N.B. \& Welbergen, J.A. (2009). Social transmission of a host defense against cuckoo parasitism. Science, 324, 1318-1320.

De Marsico, M.C., Gantchoff, M.G. \& Reboreda, J.C. (2012). Host-parasite coevolution beyond the nestling stage? Mimicry of host fledglings by the specialist screaming cowbird. Proc. R. Soc. B-Biol. Sci., 279, 3401-3408.

Feeney, W.E. \& Langmore, N.E. (2013). Social learning of a brood parasite by its host. Biology letters, 9, 20130443.

Feeney, W.E., Troscianko, J., Langmore, N.E. \& Spottiswoode, C.N. (2015). Evidence for aggressive mimicry in an adult brood parasitic bird, and generalized defences in its host. Proceedings of the Royal Society of London B: Biological Sciences, 282, 20150795.

Feeney, W.E., Welbergen, J.A. \& Langmore, N.E. (2012). The frontline of avian brood parasite-host coevolution. Anim. Behav., 84, 3-12.

Feeney, W.E., Welbergen, J.A. \& Langmore, N.E. (2014). Advances in the study of coevolution between avian brood parasites and their hosts. Annu. Rev. Ecol. Syst., 45, 227-246.

Fossøy, F., Sorenson, M.D., Liang, W., Ekrem, T., Moksnes, A., Møller, A.P., et al. (2016). Ancient origin and maternal inheritance of blue cuckoo eggs. Nat Commun, 7, 10272.

Hale, K. \& Briskie, J. (2007). Response of introduced European birds in New Zealand to experimental brood parasitism. J. Avian Biol., 38, 198-204.

Hauber, M.E. \& Sherman, P.W. (2001). Self-referent phenotype matching: theoretical considerations and empirical evidence. Trends Neurosci., 24, 609-616. 
248 Hobson, K.A. \& Villard, M.A. (1998). Forest fragmentation affects the behavioral response

249 of American Redstarts to the threat of cowbird parasitism. Condor, 100, 389-394.

250 Lahti, D.C. (2006). Persistence of egg recognition in the absence of cuckoo brood parasitism:

251 pattern and mechanism. Evolution, 60, 157-168.

252 Langmore, N.E., Cockburn, A., Russell, A.F. \& Kilner, R.M. (2009). Flexible cuckoo chick-

253 rejection rules in the superb fairy-wren. Behav. Ecol., 20, 978-984.

254 Langmore, N.E., Feeney, W.E., Crowe-Riddell, J., Luan, H., Louwrens, K.M. \& Cockburn,

255 A. (2012). Learned recognition of brood parasitic cuckoos in the superb fairy-wren

256 Malurus cyaneus. Behavioral Ecology, 23, 798-805.

257 Langmore, N.E., Hunt, S. \& Kilner, R.M. (2003). Escalation of a coevolutionary arms race

258 through host rejection of brood parasitic young. Nature, 422, 157-160.

259 Lindholm, A.K. \& Thomas, R.J. (2000). Differences between populations of reed warblers in 260 defences against brood parasitism. Behaviour, 137, 25-42.

261 Lotem, A., Nakamura, H. \& Zahavi, A. (1995). Constraints on egg discrimination and 262 cuckoo-host co-evolution. Anim. Behav., 49, 1185-1209.

263 Møller, A. (2002). Parent-offspring resemblance in degree of sociality in a passerine bird.

264 Behav Ecol Sociobiol, 51, 276-281.

265 Peer, B.D., Kuehn, M.J., Rothstein, S.I. \& Fleischer, R.C. (2011). Persistence of host defence

266 behaviour in the absence of avian brood parasitism. Biol. Lett., 7, 670-673.

267 Phillips, B.L. \& Shine, R. (2004). Adapting to an invasive species: Toxic cane toads induce

268 morphological change in Australian snakes. Proc Natl Acad Sci U S A, 101, 17150-

$269 \quad 17155$.

270 Rothstein, S.I. (1974). Mechanisms of avian egg recognition: possible learned and innate

$271 \quad$ factors. Auk, 91, 796-807. 
272 Soler, M. (2011). Could egg rejection behaviour be transmitted by social learning? Anim. 273 Behav., 81, e1-e6.

274 Soler, M. (2014). Long-term coevolution between avian brood parasites and their hosts.

275 Biological Reviews, 89, 688-704.

276 Spottiswoode, C.N. \& Stevens, M. (2012). Host-parasite arms races and rapid changes in bird 277 egg appearance. Am. Nat., 179, 633-648.

278 Stevens, M., Troscianko, J. \& Spottiswoode, C.N. (2013). Repeated targeting of the same 279 hosts by a brood parasite compromises host egg rejection. Nat. Commun., 4, 2475.

280 Su, T., Huo, J., Yang, C. \& Liang, W. (2017). Brood parasitism on barn swallow (Hirundo rustica) populations in China by common cuckoo (Cuculus canorus). Chinese Journal of Zoology, 52, 338-341.

Thompson, J.N. (1999). The evolution of species interactions. Science, 284, 2116-2118.

Thornton, A. \& Raihani, N.J. (2008). The evolution of teaching. Animal Behaviour, 75, 1823-1836.

Thorogood, R. \& Davies, N.B. (2016). Combining personal with social information facilitates host defences and explains why cuckoos should be secretive. Sci Rep, 6. discrimination in cuckoo hosts. Anim. Cogn., 18, 1373-1377.

FIGURES

295 Figure 1. Schematic outlining the experimental design (A): in the transmission trial the focal 296 received a model egg in its nest after the partner nest had rejected a model egg $(n=52)$; in the 
297 non-transmission trial the focal nest received a model egg after the partner nest had accepted

298 a model egg $(n=56)$; and in the control trial the focal nest received a model egg but the focal

299 nest had not previously received a model egg $(n=41)$. Rejection and acceptance rates in the

300 transmission, non-transmission and control trials (B). Egg rejection rates across time periods

301 by birds in focal nests across the transmission, non-transmission and control trials (C). 
